\title{
Analysis of mechanical properties for two different structures of photovoltaic pavement unit block
}

(i) The corrections made in this section will be reviewed and approved by journal production editor.

Bochao Zhou ${ }^{\mathrm{a}}$, Jianzhong Pei ${ }^{\mathrm{a}}$ peijianzhong@126.com, Ben Richard Hughes ${ }^{\mathrm{b}}$ b.hughes@strath.ac.uk, Diana SNM Nasir ${ }^{\mathrm{b}}$, Jiupeng Zhang ${ }^{\mathrm{a}}$

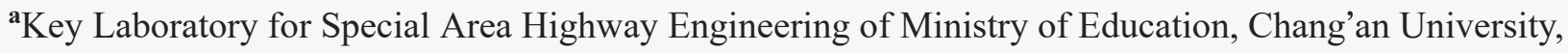
Xi'an, Shaanxi 710064, China

${ }^{\mathbf{b}}$ Department of Aerospace and Mechanical Engineering, University of Strathclyde, Glasgow, Scotland G1 1XQ, United Kingdom

*Corresponding authors.

\begin{abstract}
Recently, photovoltaic (PV) pavement has widely attracted attention as an alternative to provide renewable energy. Research which focuses on the mechanical properties of a PV pavement is still at an early stage of exploration. This study adopted two types of PV pavement unit block structure, namely grid unit block and hollow unit block based on previous literature. ABS was selected as the material of the unit block body, which is proven has strong mechanical properties and can be recycled. Effects of various factors on the mechanical properties of the unit blocks were analysed, including (i) structure length, (ii) structure width, (iii) thickness of bottom plate and (iv) thickness of grid or wall. Orthogonal test was used to obtain 16 sets of experiment for each unit block structure and numerical simulation was conducted by ABAQUS. The surface longitudinal deformation $l_{s}$ and the maximum tensile stress $\sigma_{m}$ at the centre of the bottom of the lighttransmitting plate are the main indexes for mechanical response analysis. Mean analysis was used to determine the optimal combination of the structural sizes, meanwhile multivariate analysis of variance was used to rank the significance of each factor. Results have demonstrated that the optimal size combinations for the two structures are: (i) for grid unit block structure $-120 \mathrm{~cm}$ length $\times 120 \mathrm{~cm}$ width $\times 8 \mathrm{~cm}$-thickness bottom plate $\times 2 \mathrm{~cm}$-thickness grid (ii) for hollow unit block structure $-60 \mathrm{~cm}$ length $\times 60 \mathrm{~cm}$ width $\times 6 \mathrm{~cm}$-thickness bottom plate $\times 10 \mathrm{~cm}$-thickness side walls. Meanwhile, the ranks significance of each factor are: (i) for grid unit block structure -
\end{abstract}


plate thickness $>$ grid thickness $>$ width $>$ length (ii) for hollow unit block structure - only width is

significant to $\sigma_{m}$. The results also suggested that grid unit block structure for a PV pavement is better than hollow unit block structure.

Keywords: Photovoltaic pavement; Mechanical properties; Surface longitudinal deformation $l_{s}$; Maximum tensile stress $\sigma_{m}$; Optimal combination; Factor significance

\section{Introduction and literature review}

Energy and environment are the two important aspects for sustainability which recently become the topics of interest among various research fields, especially towards the development and the utilisation of energy saving and renewable energy. More than a decade, roads as infrastructure facilities have paved its contribution towards making in more sustainable in terms of its construction, development and function [1-4].

In constructing road energy system, several techniques are studied in generating energy on roads, which are piezoelectricity $[5,6]$, thermoelectricity $[7,8]$ and photoelectricity $[9,10]$. Piezoelectricity technique allows the energy to be converted based on the generated vibration from the vehicle movement. Meanwhile, thermoelectricity and photoelectricity techniques utilise solar radiation as the main source of energy to be generated in terms of thermal and electricity, respectively. Among the three techniques, the application of PV pavement is the most recent and has been seen with great potential as it can produce massive energy from the abundant solar and is clean to environment [11]. Simultaneously, solar radiation also worsens the asphalt aging and urban heat island effect. Therefore, based on energy conservation law, high thermal effect on roads can be lessen by converting the absorbed solar into a useful energy [12].

In 2009, Brusaw proposed and designed an interlocking hexagonal PV solar panel as a future road pavement in replacing the conventional pavement [13]. By laying 1/3 of the road with solar panels, the electricity demand for entire United States was reported can be fully met. Based on the testing results on impact resistance and traction, the designed solar panel was reported to sustain up to 250,000 -pound $(\approx 113,400 \mathrm{~kg})$ load and its traction can stop a vehicle moving up to 80 miles per hour $(\approx 150 \mathrm{~km} / \mathrm{h})$ [14]. Although the cost is still becoming a challenge in making it applicable and cost effective for real use, the conceptual road solar panel has sparked more thoughts from both industrial and academic research to begin to explore the integration of PV into road pavements.

In 2012, Tighe et al. developed a solar road panel with three-level structure using numerical model. It comprises of a transparent layer (two sub-layers of 10-mm-thick tempered glass), an optical layer (two sublayers of 6.35-mm-thick GPO-3 laminate fiberglass) and a base layer with $19.05 \mathrm{~mm}$ GPO-3 laminate fiberglass (12.7-mm plate $+6.35-\mathrm{mm}$ plate) [15-17]. In the study, the thickness of each layer was proposed and each grid was designed to ensure an adequate space to slot in the $125-\mathrm{mm}$ solar cell. However, the overall size (length and width) of the solar panel was not discussed. In 2016, numerical hollow slab element structure for solar pavement was proposed by X Zha et al. Three layers were proposed for the new structure, which are Polymethyl methacrylate (PMMA) transparent protection layer, solar cells layer and prefabricated concrete hollow base layer [18]. In the study, four factors of a solar panel: (i) length, (ii) width, (iii) side walls thickness 
and (iv) PMMA thickness were analysed by using ANSYS. Based on the comprehensive results of the mechanical properties by considering safety and economic point of view, the optimised overall structure was recommended as $50 \mathrm{~cm} \times 50 \mathrm{~cm} \times 25 \mathrm{~cm}$, wherein the thickness of PMMA, side wall and baseplate are $2 \mathrm{~cm}$, $10 \mathrm{~cm}$ and $10 \mathrm{~cm}$, respectively. However, the rank of the four factors was not carried out in the study, which should be investigated to provide guidance for future system optimisation.

In considering sustainable environment, numerous researchers have highlighted on reusing waste materials for road construction, such as steel slag waste [19], glass waste [20] and plastic waste [21]. Among the three waste materials, it was reported that plastic waste has produced over 300 million tons annually [22]. Remarkably, some scholars shifted to give much concern on "plasticence" period, where people are too dependent on using plastic; therefore, re-use of the plastic waste has raised much attention and has been seen as a significant potential for saving the environment [23]. As of 2015, 6.3 billion tons of plastic waste has been produced worldwide; yet, only $9 \%$ of the total waste has been recycled [24]. Take China as an example, the annual production of plastic waste is 23 million tons and has accounted for $11 \%$ of municipal solid waste, however, the average recycling rate is only $25 \%[25,26]$. In Europe, plastic waste has been produced up to 25 million tons annually with less than $30 \%$ has been recycled [27]. Therefore, it is a priority to enhance the research on recycling plastic waste for large scale applications. Common plastic materials in daily use have included PET, PE (including HDPE and LDPE), PP, PS, PVC, PC, ABS, EVA, EPS, etc. [28]. Among these materials, ABS is the thermoplastics polymer which can be reprocessed for recycling and reusing. Also, it is widely used in manufacturing and chemical industries i.e. machinery, electrical, textile, automotive, aircraft, and shipbuilding. ABS was also reported for having strong mechanical properties, good chemical resistance, good heat resistance, high dimensional stability, good electrical insulation and is in low cost [29,30]. Compared to the conventional materials like asphalt concrete, there is harmful volatile released from asphalt when it is at a high temperature [31]. Meanwhile, the main mechanical parameters of ABS (Young's modulus and Poisson ratio) are similar. Therefore, ABS has been identified as a potential to be recycled and reused for massive development like road construction.

The current PV pavement unit structures are mainly the grid block structure and the hollow block structure according to the previous research. Therefore, the above two structures are mainly analysed in this study. The aim of this study is to investigate the mechanical properties of two different structures of PV pavement unit block and the objective of the study is to obtain the optimal structure sizes based on orthogonal test and SPSS analysis. Discrete element method (DEM) and finite element method (FEM) are the most common in the current calculation method of pavement structure [32,33]. DEM is more suitable for the microstructural calculation (micron and millimetre level) [34-36], and the FEM is more used to simulate on the macro scale structural response. Therefore, ABAQUS 6.14 was used to conduct finite element simulation in this study for analysing the four factors affecting the two structural mechanical properties mentioned above and these factors were ranked by conducting SPSS analysis. Meanwhile, ABS was proposed to be used in the two structures for PV pavement by considering the reason for plastic waste recycling and reusing and also for its having good mechanical strength.

\section{Unit block structures of PV pavement}


Based on previous literature, there are two different unit block structures for PV pavement, namely grid unit block structure and hollow unit block structure $[15,18]$. In this study, the above two structure were selected to analyse their mechanical properties by using ABAQUS.

\subsection{Grid unit block structure}

Based on the solar pavement panel which was proposed by Tighe et al. as shown in Fig. 1(a) [17], the structure layer which comprises of an optical layer and a base layer was re-designed by integrating the two layers into one layer, which is more convenient to pave. Therefore, in this study, the grid unit block structure only comprises of a transparent layer and a base layer. As the former design strived to keep the solar cells from being damaged and to ensure the light reaches the slot, the design developed for this study strived to provide an adequate slotting space to install solar cells, as shown in Fig. 1(b). In Fig. 1(b), $a$ is the length of the structure, in parallel to the driving direction; $b$ is the width of the structure, in perpendicular to the driving direction; $c$ is the thickness of light-transmitting plate; $t$ is the thickness of side walls, $h$ is the height of structure layer, $d$ is the thickness of grid; $h_{l}$ is the groove clearance for solar cells; and $h-h_{1}$ is the thickness of bottom plate.

\section{Fig. 1}

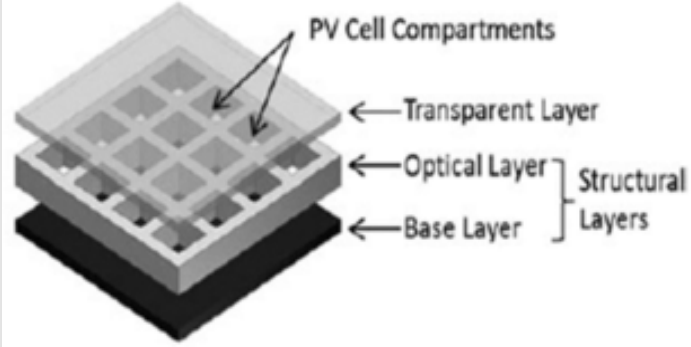

(a) Solar pavement panel from [16]

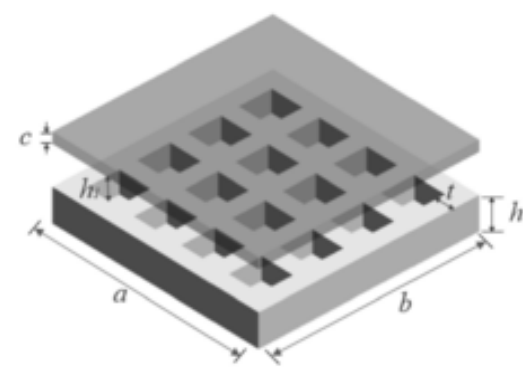

(b) Grid unit block for PV pavement

Schematic diagram of the grid unit block structure for PV pavement.

\subsection{Hollow unit block structure}

As shown in Fig. 2, the hollow unit block structure was modelled similar to the hollow slab element structure proposed by Zha et al. [18]. The structure comprises of a transparent layer and a base layer, using the same characteristics of the grid unit block structure to describe and determine the size of the hollow unit block, wherein $t$ represents the thickness of side wall. 


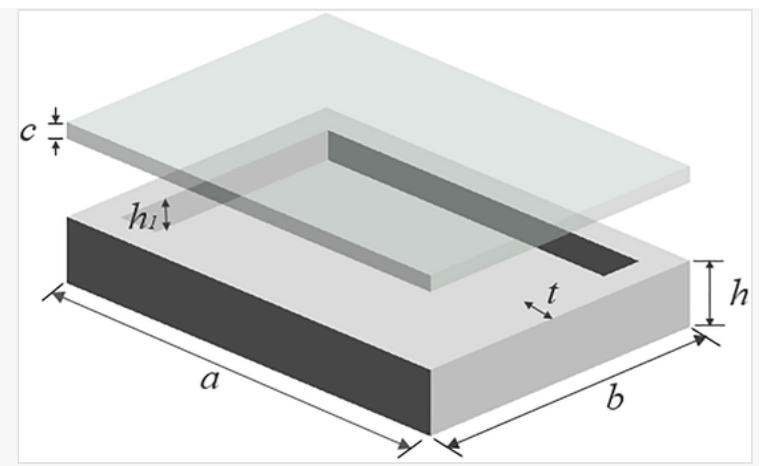

Schematic diagram of the hollow unit block structure for PV pavement.

\section{Mechanical analysis of the unit block structures for PV pavement}

ABAQUS is an engineering finite element simulation software which can solve a large number of structural (stress/displacement) problems. Therefore, ABAQUS is selected for numerical simulation analysis in this study.

To simplify the stress analysis of the hollow unit block, the model was regarded as a single hollow unit block on the homogeneous base. Therefore, the following assumptions were used:

(1) The interlayer contact (a) between the bottom surface of unit blocks and foundation and (b) between the top surface of unit blocks and the light-transmitting plate are completely continuous;

(2) For the foundation, the base layer and the subgrade are considered to be equivalent homogeneous and are characterised by the equivalent rebound modulus of the top surface of the base layer;

(3) The base plate, the light-transmitting plate and the foundation are regarded as uniform, continuous and isotropic linear elastic bodies;

(4) The transfer effect between multiple plates is not being considered;

(5) The self-weight of the whole structure is excluded.

To establish the model, the base bottom was fully constrained and the surrounding sides which perpendicular to the base bottom were applied with a normal constraint. The surrounding sides of the unit blocks were unconstrained and were set with free edges. As refer to the results of the convergence analysis by [18], the foundation depth was set to $5.0 \mathrm{~m}$ and the base surface had been extended outwardly by $1.5 \mathrm{~m}$ from the longitudinal and horizontal edges of the unit blocks. The BZZ-100 standard axle load of the model was set to be equivalent to double circular uniform loading with $0.7 \mathrm{MPa}$ ground pressure, meanwhile the diameter of each round and the distance at the centre was set $213 \mathrm{~mm}$ and $319.5 \mathrm{~mm}$, respectively, following the work of [37].

Since only the unit block structures were analysed, the load at the edge of the unit blocks was not considered, which should be considered if analysing the transmission of load between unit blocks. The analysis results 
based on $[17,38]$ suggested that the most unfavourable position of the load is the symmetrical position at the centre of the unit blocks; therefore, only the centre load was tested in this study. The main indexes for mechanical response analysis are the surface longitudinal deformation $l_{s}$ and the maximum tensile stress $\sigma_{m}$ at the centre of the bottom of the light-transmitting plate. Further adoptions to the unit blocks are: (i) solid element model adopted the default unit type C3D8R, (ii) the interlayer contact adopted the face-to-face contact method, (iii) the unit division for unit blocks was set as $10 \mathrm{~mm}$, and (iv) the base adopted the method of gradually encrypted from bottom to top.

\subsection{Material properties}

ABS was proposed as the main material for the base layer of the unit blocks. Using ABS is an alternative solution based on the reuse of plastic waste for saving the environment and simultaneously it provides better endurance for PV units to be integrated with road pavement [39]. In compared to cement concrete which consumes non-renewable natural resources i.e. stone [18], recycle and reuse ABS as road materials indirectly become a sustainable construction approach for future road energy system.

The important requirements for a PV pavement are: (i) high transparency, (ii) high strength, and (iii) only allows minor deformation. Therefore, the tempered glass was applied as the transparent layer of the PV units $[10,17]$. The materials and the parameters set for the PV unit blocks are detailed in Table 1.

Table 1

i) The presentation of Tables and the formatting of text in the online proof do not match the final output, though the data is the same. To preview the actual presentation, view the Proof.

Materials and parameters of unit blocks for PV pavement.

\begin{tabular}{|c|c|c|c|}
\hline \multirow[b]{2}{*}{ Part } & \multirow[b]{2}{*}{ Materials } & \multicolumn{2}{|l|}{ Parameters } \\
\hline & & Young's modulus(MPa) & Poisson ratio \\
\hline Foundation & / & 200 & 0.3 \\
\hline Structure layer & ABS & 2000 & 0.4 \\
\hline Transparent layer & Tempered glass & 72,000 & 0.2 \\
\hline
\end{tabular}

\subsection{Orthogonal test design and results}

Analysis using controlled variates method was carried out in the study of Zha et al. to discuss the mechanical properties of the hollow structure [18]. When the study analysed the influence law of a certain parameter change on the mechanical response, other parameters were uniformly selected. The study only considered the analysis based on one changing parameter while ignoring the effects of other changing parameters. Furthermore, the meaning of the factors for length and width of the hollow structure were not explicitly 
described. Therefore, the recommended size combination may not be the optimum size considering only one parameter. In order to obtain more reasonable optimal combination to determine the structural size, an orthogonal test design was used in this study. Four factors of sizing the grid unit and hollow unit block structures were considered in this study based on the following parameters: (i) structure length, $a$ (ii) structure width, $b$, (iii) thickness of bottom plate, $h-h_{1}$ and (iv) thickness of grid or wall, $t$. The calculation parameters with four value levels are described in Tables 2 and 3 for grid unit block structure and hollow unit block structure, respectively. It is noted that the length of the structure is parallel to the vehicle driving direction meanwhile the width of the structure is perpendicular to the vehicle driving direction.

Table 2

(i) The presentation of Tables and the formatting of text in the online proof do not match the final output, though the data is the same. To preview the actual presentation, view the Proof.

Taking value of calculation parameters for grid unit block structure.

\begin{tabular}{|c|c|c|c|c|c|}
\hline \multirow{2}{*}{ Parameter } & & \multicolumn{4}{|c|}{ Value level } \\
\hline & & 1 & 2 & 3 & 4 \\
\hline Length $\mathrm{a} / \mathrm{cm}$ & A & 60 & 80 & 100 & 120 \\
\hline Width $b / \mathrm{cm}$ & B & 60 & 80 & 100 & 120 \\
\hline Bottom plate thickness $h-h_{1} / \mathrm{cm}$ & $\mathrm{C}$ & 2 & 4 & 6 & 8 \\
\hline Grid thickness $t / \mathrm{cm}$ & $\mathrm{D}$ & 1 & & 2 & \\
\hline Tempered glass thickness $c / \mathrm{cm}$ & / & 2 & & & \\
\hline Grid clearance $h_{1} / \mathrm{cm}$ & / & 2 & & & \\
\hline
\end{tabular}

Note: The bold is the different parameters and value level for the two structures.

Table 3

(i) The presentation of Tables and the formatting of text in the online proof do not match the final output, though the data is the same. To preview the actual presentation, view the Proof.

Taking value of calculation parameters for hollow unit block structure.

\begin{tabular}{|l|l|l|l|l|l|}
\hline Parameter & \multicolumn{3}{c|}{ Value level } \\
& & $\mathbf{1}$ & $\mathbf{2}$ & $\mathbf{3}$ & $\mathbf{4}$ \\
\hline Length $a / \mathrm{cm}$ & A & 60 & 80 & 100 & 120 \\
\hline
\end{tabular}




\begin{tabular}{|l|l|l|l|l|l|}
\hline Width $b / \mathrm{cm}$ & B & 60 & 80 & 100 & 120 \\
\hline Bottom plate thickness $h-h_{1} / \mathrm{cm}$ & C & 4 & 6 & 8 & 10 \\
\hline Side walls thickness $t / \mathrm{cm}$ & D & $\mathbf{4}$ & $\mathbf{6}$ & $\mathbf{8}$ & $\mathbf{1 0}$ \\
\hline Tempered glass thickness $c / \mathrm{cm}$ & $/$ & 2 & $/$ & 2 & \\
\hline Hollow clearance $h_{l} / \mathrm{cm}$ & & 2 &
\end{tabular}

Note: The bold is the different parameters and value level for the two structures.

If all combinations were considered, there would be 128 combinations and 256 combinations for grid unit structure and for hollow unit structure, respectively. In order to reduce the amount of tests, orthogonal test design method was adopted based on statistics and the orthogonality principle. Orthogonal test design is a multi-factor and multi-level design method, which relies on the orthogonality of the orthogonal table to select some representative points from the comprehensive test for testing. This can achieve the equivalent of a large number of comprehensive tests with the minimum number of test. As it is a high efficiency, fast and economical experimental design method, it was used to design the tests for this study.

For grid unit block structure, the orthogonal test design of 3 factors with 4 levels and 1 factor with 2 levels was adopted. Therefore, the amount of test was reduced to 16 tests. As the most common size for solar cell is $15.6 \mathrm{~cm} \times 15.6 \mathrm{~cm}$, the grid layout was determined to ensure an adequate space to embed the solar cells. The orthogonal list and results are shown in Table 4.

\section{Table 4}

(i) The presentation of Tables and the formatting of text in the online proof do not match the final output, though the data is the same. To preview the actual presentation, view the Proof.

Orthogonal test design and numerical simulation resultsfor grid unit block structure.

\begin{tabular}{|c|c|c|c|c|c|c|c|}
\hline No. & $a / \mathrm{cm}$ & $b / \mathrm{cm}$ & $h-h_{1} / \mathrm{cm}$ & $t / \mathrm{cm}$ & $l_{s} / \mathrm{mm}$ & $\sigma_{m} / \mathbf{M P a}$ & Grid layout \\
\hline 1 & 80 & 80 & 2 & 2 & 0.59 & 8.83 & $4 \times 4$ \\
\hline 2 & 100 & 100 & 2 & 2 & 0.60 & 12.58 & $5 \times 5$ \\
\hline 3 & 120 & 120 & 2 & 1 & 0.63 & 9.55 & $6 \times 6$ \\
\hline 4 & 120 & 100 & 8 & 1 & 0.51 & 8.83 & $6 \times 5$ \\
\hline 5 & 60 & 100 & 4 & 2 & 0.56 & 11.71 & $3 \times 5$ \\
\hline 6 & 100 & 60 & 4 & 1 & 0.65 & 13.47 & $5 \times 3$ \\
\hline 7 & 100 & 120 & 8 & 2 & 0.49 & 9.96 & $5 \times 6$ \\
\hline 8 & 60 & 80 & 8 & 1 & 0.57 & 10.87 & $3 \times 4$ \\
\hline
\end{tabular}




\begin{tabular}{|l|l|l|l|l|l|l|l|}
\hline 9 & 120 & 80 & 4 & 2 & 0.54 & 8.06 & $6 \times 4$ \\
\hline 10 & 120 & 60 & 6 & 2 & 0.53 & 8.08 & $6 \times 3$ \\
\hline 11 & 80 & 120 & 4 & 1 & 0.59 & 8.80 & $4 \times 6$ \\
\hline 12 & 80 & 60 & 8 & 2 & 0.51 & 7.56 & $4 \times 3$ \\
\hline 13 & 60 & 120 & 6 & 2 & 0.53 & 10.17 & $3 \times 6$ \\
\hline 14 & 80 & 100 & 6 & 1 & 0.55 & 8.82 & $4 \times 5$ \\
\hline 15 & 100 & 80 & 6 & 1 & 0.59 & 11.49 & $5 \times 4$ \\
\hline 16 & 60 & 60 & 2 & 1 & 0.69 & 14.09 & $3 \times 3$ \\
\hline
\end{tabular}

For hollow unit block structure, the orthogonal test design of 4 factors with 4 levels was adopted. Therefore, the total 16 tests were carried out; see the orthogonal list and results as shown in Table 5 below.

\section{Table 5}

(i) The presentation of Tables and the formatting of text in the online proof do not match the final output, though the data is the same. To preview the actual presentation, view the Proof.

Orthogonal test design and numerical simulation results for hollow unit block structure.

\begin{tabular}{|c|c|c|c|c|c|c|}
\hline NO. & $a / \mathrm{cm}$ & $b / \mathrm{cm}$ & $h-h_{1} / \mathrm{cm}$ & $t / \mathrm{cm}$ & $l_{s} / \mathrm{mm}$ & $\sigma_{m} / \mathrm{MPa}$ \\
\hline 1 & 120 & 60 & 10 & 10 & 1.86 & 25.03 \\
\hline 2 & 60 & 60 & 4 & 4 & 1.71 & 26.50 \\
\hline 3 & 120 & 120 & 8 & 6 & 7.73 & 53.01 \\
\hline 4 & 60 & 120 & 6 & 8 & 1.69 & 31.61 \\
\hline 5 & 100 & 60 & 8 & 8 & 1.26 & 22.25 \\
\hline 6 & 60 & 80 & 8 & 10 & 1.18 & 23.99 \\
\hline 7 & 120 & 100 & 4 & 8 & 5.23 & 44.09 \\
\hline 8 & 80 & 100 & 8 & 4 & 4.43 & 45.16 \\
\hline 9 & 120 & 80 & 6 & 4 & 4.48 & 40.70 \\
\hline 10 & 100 & 100 & 6 & 10 & 3.80 & 39.81 \\
\hline 11 & 80 & 80 & 10 & 8 & 2.23 & 33.59 \\
\hline 12 & 80 & 60 & 6 & 6 & 1.54 & 23.47 \\
\hline 13 & 60 & 100 & 10 & 6 & 1.97 & 33.32 \\
\hline
\end{tabular}




\begin{tabular}{|l|l|l|l|l|l|l|}
\hline 14 & 100 & 80 & 4 & 6 & 3.38 & 36.96 \\
\hline 15 & 100 & 120 & 10 & 4 & 7.24 & 53.83 \\
\hline 16 & 80 & 120 & 4 & 10 & 3.06 & 40.10 \\
\hline
\end{tabular}

\section{Result and discussion}

In this study, ABAQUS was used for numerical simulation meanwhile Statistical Product and Service Solutions (SPSS) was used to analyse the data. SPPS is a software which performs a wide range of statistical procedures. The basic functions of SPSS include the data management, statistical analysis, chart analysis, output management and etc. For statistical analysis, it processes the descriptive statistics, mean comparison, general linear model, correlation analysis and etc. Descriptive analysis is a basic description of the data, which mainly used to describe the basic characteristics of the variables. It can generate relevant descriptive statistics such as mean, variance, standard deviation, full range, kurtosis and skewness. Through these descriptive statistics, we can fully understand the comprehensive characteristics of the variables. In this study, the mean analysis and variance analysis were adopted. Mean analysis was used to gain the optimal level combination for each unit block structure, meanwhile variance analysis was used to analyse whether the difference is significant for the different factors. Based on the result of variance analysis, the importance of the factors was ranked.

\section{$4.1 \sigma$ - and $l$ - contours of the unit blocks}

In order to clearly observe the stress and strain of grid and hollow unit blocks, the base part was hidden while only the $\sigma_{m}$ - and $l_{s}$-contours of the unit blocks sliced at the middle section based on width direction were shown, see Fig. 3. For $\sigma_{m}$-contours, hot colour contours represent tensile stress value, meanwhile the cold colour contours represent compressive stress value. And for $l_{s}$-contours, the hot colour contours represent small deformation value, meanwhile the cold colour contours represent high deformation value, due to the direction of longitudinal deformation is down. Example of $\sigma_{m}$-contours and $l_{s}$-contours of the grid and hollow unit block structures were shown by selecting the design no 16, 2 and 13 for grid unit block and design no 2, 10 and 4 for hollow unit block. For both types of unit block, the examples were selected in order to show the distribution of tensile stress and longitudinal deformation clearly, and according to the same value of length (a) and width $(b)$.

\section{Fig. 3}




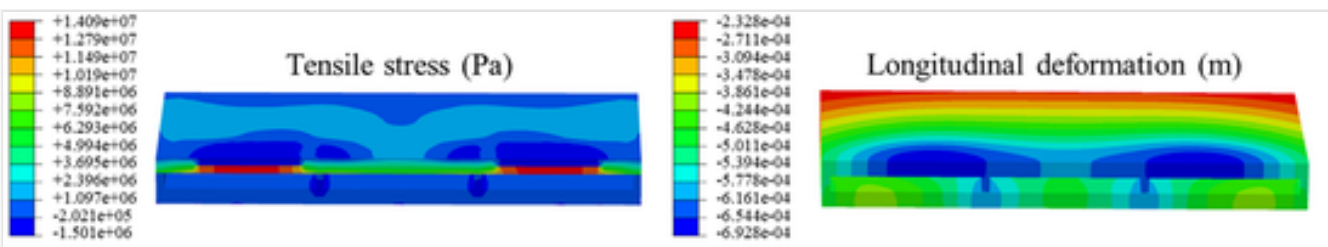

Grid unit block, No. $16, a: 60 \mathrm{~cm}, b: 60 \mathrm{~cm}, h-h_{l}: 2 \mathrm{~cm}, t: 1 \mathrm{~cm}$

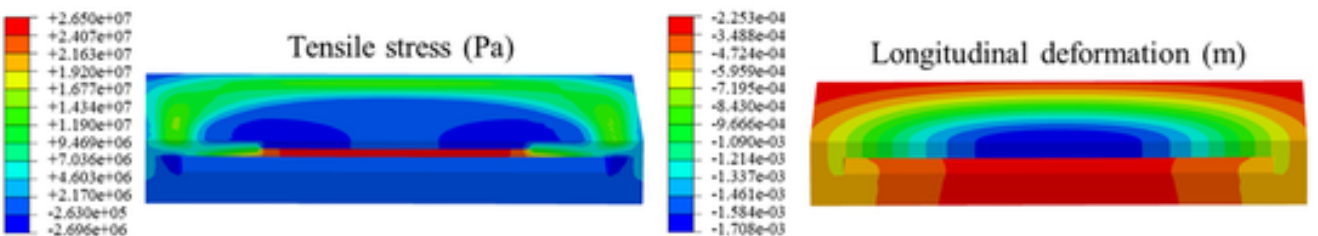

Hollow unit block, No. $2, a: 60 \mathrm{~cm}, b: 60 \mathrm{~cm}, h-h_{l}: 4 \mathrm{~cm}, t: 4 \mathrm{~cm}$

(a)
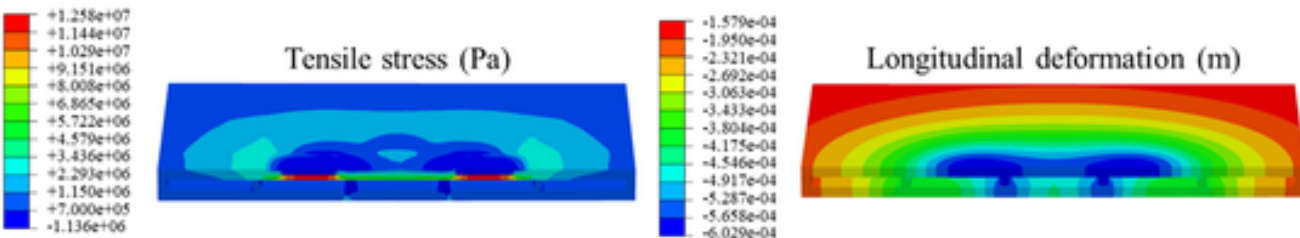

Grid unit block, No. 2, $a: 100 \mathrm{~cm}, b: 100 \mathrm{~cm}, h-h_{l}: 2 \mathrm{~cm}, t: 2 \mathrm{~cm}$
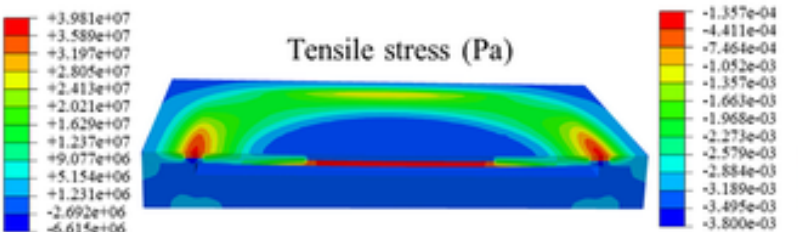

Longitudinal deformation $(\mathrm{m})$

Hollow unit block, No. $10, a: 100 \mathrm{~cm}, b: 100 \mathrm{~cm}, h-h_{l}: 6 \mathrm{~cm}, t: 10 \mathrm{~cm}$

(b)
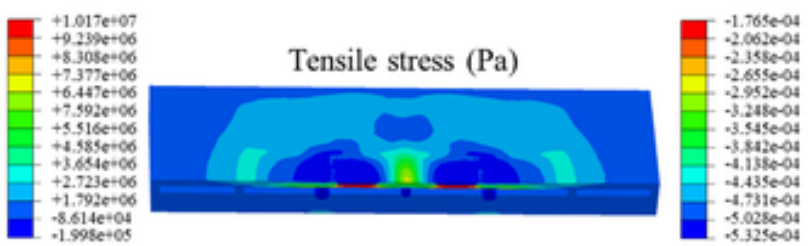

Longitudinal deformation (m)

Grid unit block, No. $13, a: 60 \mathrm{~cm}, b: 120 \mathrm{~cm}, h-h_{l}: 6 \mathrm{~cm}, t: 2 \mathrm{~cm}$
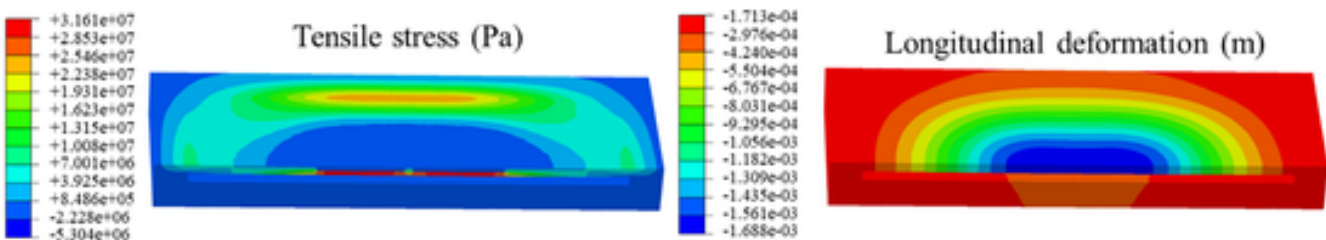

Hollow unit block, No. $4, a: 60 \mathrm{~cm}, b: 120 \mathrm{~cm}, h-h_{l}: 6 \mathrm{~cm}, t: 8 \mathrm{~cm}$

(c)

Structure contours for grid unit block and hollow unit block.

Based on the results of $\sigma_{m}$ and $l_{s}$, the SPSS was used to analyse further by importing the data of Tables 4 and 5.

\subsection{Mean analysis}


In this study, mean analysis was used to explore the optimal combination of structural sizes and the results for grid unit block structure are shown in Fig. 4. The ultimate tensile strength of tempered glass was 82Mpa with the structural safety factor of 1.5, meanwhile the corresponding allowable tensile stress control value was $55 \mathrm{Mpa}$. The result of maximum tensile stress $\sigma_{m}$ from the simulation was significantly smaller as compared to the allowable tensile strength; therefore, the $\sigma_{m}$ is safe enough to the structure. In order to make sure the grid clearance is adequate for embedding solar cells while the driving comfort was considered, $l_{s}$ was taken as the main factor for the safety of the structure. Based on the mean analysis of four different factors with multilevels as shown in Fig. 4, the optimal combination value was A4B4C4D2, given the total dimension of $120 \mathrm{~cm}$ length $\times 120 \mathrm{~cm}$ width $\times 8 \mathrm{~cm}$-thickness bottom plate $\times 2 \mathrm{~cm}$-thickness grid.

\section{Fig. 4}

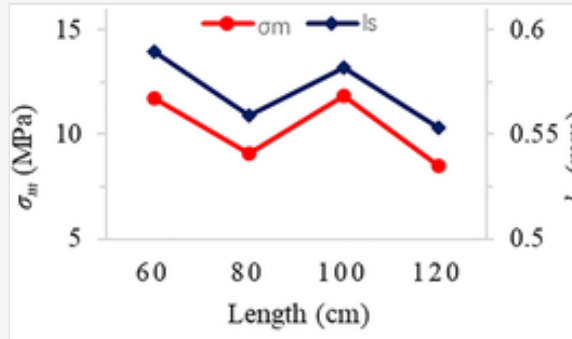

(a)

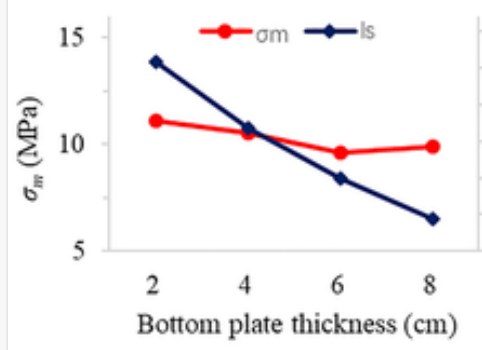

(c)

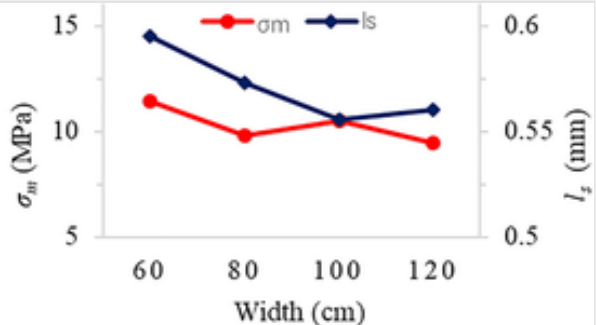

(b)

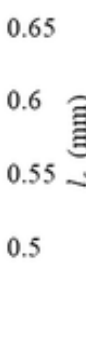

0.6

.5
11

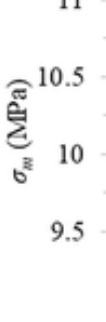

Relation curves of $l_{s}$ and $\sigma_{m}$ with each parameter for grid unit block structure.

For grid unit block structure, results have significantly shown that the mechanical properties fluctuated along with the value of length and width. However, when compared the four value levels, the smallest $l_{s}$ was at the largest value of length and width, see Fig. 4(a) and (b). Therefore, a contrast conclusion was drawn to compare with the study of Zha et al. [18] by suggesting the smallest value of the length and the width as optimum to achieve the best structure. By suggesting this, the whole size of the unit block may not be limited to a certain size of PV pavement, which means the unit block can be designed as large as the cement pavement panel. The construction process of cement pavement can be improved by replacing it to the PV pavement, which expedites the development of PV pavement.

The thickness of the bottom plate and the grid also influenced the mechanical properties of the structure and the $l_{s}$ value decreases according to the increase value of the bottom plate and the grid, see Fig. 4(c) and (d). For these two factors, the best value was suggested $8 \mathrm{~cm}$ and $2 \mathrm{~cm}$ for the bottom plate and the grid, respectively. 
Similar analysis was conducted for hollow unit block structure and the results of mean analysis is shown in Fig. 5. For hollow unit block structure, $l_{s}$ and $\sigma_{m}$ were both considered as the $\sigma_{m}$ value is closed to the allowable tensile stress. The trends for $\sigma_{m}$ and $l_{s}$ were found similar and the results in Fig. 5 suggested that the optimal combination value was A1B1C3D3, given the total dimension of $60 \mathrm{~cm}$ length $\times 60 \mathrm{~cm}$ width $\times 6 \mathrm{~cm}$-thickness bottom plate $\times 10 \mathrm{~cm}$-thickness side walls.

\section{Fig. 5}

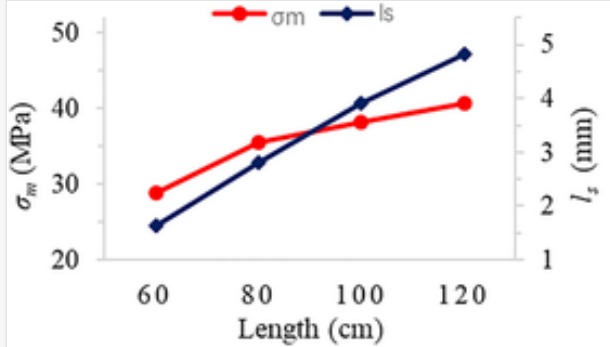

(a)

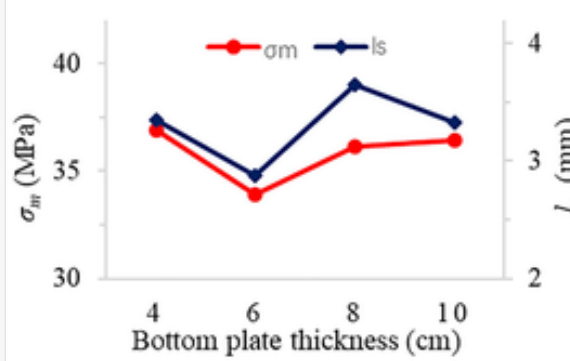

(c)

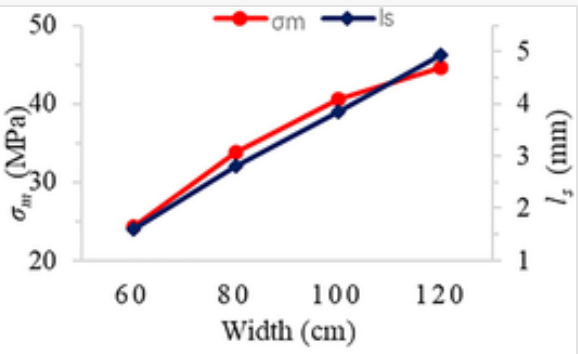

(b)

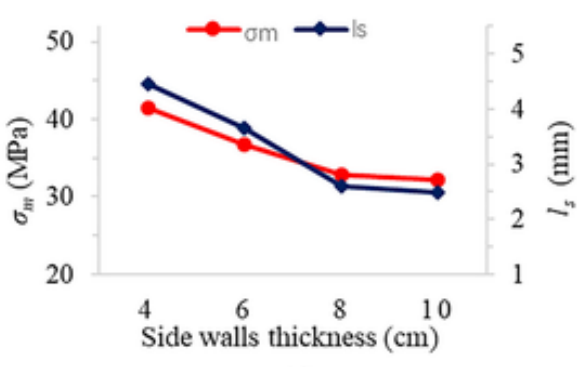

(d)

Relation curves of $l_{s}$ and $\sigma_{m}$ with each parameter for hollow unit block structure.

Based on the findings, the optimal combination value was shown similar to the work of Zha et al. [18], which an optimum structure can be achieved with smallest value of length and width, with the thickest bottom plate and with the thickest side walls, see Fig. 5. However, a dissimilar trend was observed from the effect of bottom plate thickness on mechanical properties, derived from the fluctuation values shown in the graph result, see Fig. 5(c). Another study has drawn a conclusion with insignificant effect of bottom plate thickness on the mechanical properties of the hollow unit block structure [40].

\subsection{Multivariate analysis of variance}

Multivariate analysis of variance is used to study whether a dependent variable is affected by multiple independent variables (factors) and to test whether there is a significant difference for the mean values of the dependent variables among different combinations of the value of the factors. In this study, multivariate analysis of variance was used to analyse the main effects of different factors on $l_{s}$ and $\sigma_{m}$.

For the grid unit block structure, the orthogonal test results of multivariate analysis of variance for $l_{s}$ and $\sigma_{m}$ are shown in Tables 6 and 7. 
(i) The presentation of Tables and the formatting of text in the online proof do not match the final output, though the data is the same. To preview the actual presentation, view the Proof.

Orthogonal test results of multivariate analysis of variance of $\sigma_{m}$ for grid unit block structure.

\begin{tabular}{|l|l|l|l|l|l|}
\hline Variance source & Sum of square & Degree of freedom & Mean square & F value & P value \\
\hline Length $(\mathrm{cm})$ & 36.977 & 3 & 12.326 & 53.265 & $3.23 \mathrm{E}-4$ \\
\hline Width $(\mathrm{cm})$ & 8.741 & 3 & 2.914 & 12.591 & $9.111 \mathrm{E}-3$ \\
\hline Bottom plate thickness $(\mathrm{cm})$ & 5.143 & 3 & 1.714 & 7.408 & $2.7444 \mathrm{E}-2$ \\
\hline Grid thickness $(\mathrm{cm})$ & 2.207 & 1 & 2.207 & 9.536 & $2.7219 \mathrm{E}-2$ \\
\hline Deviation & 1.157 & 5 & 16 & & \\
\hline Total & 1748.041 & 16 & & \\
\hline
\end{tabular}

\section{Table 7}

(i) The presentation of Tables and the formatting of text in the online proof do not match the final output, though the data is the same. To preview the actual presentation, view the Proof.

Orthogonal test results of multivariate analysis of variance of $l_{S}$ for grid unit block structure.

\begin{tabular}{|c|c|c|c|c|c|}
\hline Variance source & Sum of square & Degree of freedom & Mean square & F value & $P$ value \\
\hline Length $(\mathrm{cm})$ & 0.004 & 3 & 0.001 & 65.761 & $1.94 \mathrm{E}-4$ \\
\hline Width $(\mathrm{cm})$ & 0.004 & 3 & 0.001 & 66.757 & $1.87 \mathrm{E}-4$ \\
\hline Bottom plate thickness $(\mathrm{cm})$ & 0.026 & 3 & 0.009 & 453.842 & $2 \mathrm{E}-6$ \\
\hline Grid thickness (cm) & 0.010 & 1 & 0.010 & 532.170 & $3 \mathrm{E}-6$ \\
\hline Deviation & $9.438 \mathrm{E}-5$ & 5 & & & \\
\hline Total & 5.260 & 16 & & & \\
\hline
\end{tabular}

Based on Tables 6 and 7, all four factors tested on $\sigma_{m}$ and $l_{s}$ have significantly influenced on both unit structures. Findings suggested that the significance of the four factors for the $\sigma_{m}$ was ranked as follows: A (length) $>\mathrm{B}$ (width) $>\mathrm{D}$ (grid thickness) $>\mathrm{C}$ (bottom plate thickness). Meanwhile, the significance of the four factors for $l_{s}$ was ranked as follow: $\mathrm{C}$ (bottom plate thickness) $>\mathrm{D}$ (grid thickness) $>\mathrm{B}$ (width) $>\mathrm{A}$ (length). 
Remarkably, the rank of significance for the four factors comparing $\sigma_{m}$ and $l_{s}$ were shown opposite. In this study, from the mean analysis, the result of $\sigma_{m}$ from the simulation was significantly smaller as compared to the allowable tensile strength, the $l_{s}$ was favourably taken into account. Therefore, the bottom plate thickness is the most important to the grid unit block structure. The rank of these factors can provide reference for the future work of the structure for PV pavement, which is not be considered before. By combining the variance analysis for mechanical properties and considering to reduce the cost by saving material, the optimal combination of grid unit block structure was adjusted to A4B4C.

For the hollow unit block structure, the orthogonal test results of multivariate analysis of variance for $l_{s}$ and $\sigma_{m}$ are shown in Tables 8 and 9.

(i) The presentation of Tables and the formatting of text in the online proof do not match the final output, though the data is the same. To preview the actual presentation, view the Proof.

Orthogonal test results of multivariate analysis of variance of $\sigma_{m}$ for hollow unit block structure.

\begin{tabular}{|l|l|l|l|l|l|}
\hline Variance source & Sum of square & Degree of freedom & Mean square & F value & P value \\
\hline Length $(\mathrm{cm})$ & 312.717 & 3 & 104.239 & 7.567 & 0.065 \\
\hline Width $(\mathrm{cm})$ & 948.041 & 3 & 316.014 & 22.941 & 0.014 \\
\hline Side walls thickness $(\mathrm{cm})$ & 220.177 & 3 & 73.392 & 5.328 & 0.101 \\
\hline Bottom plate thickness $(\mathrm{cm})$ & 21.422 & 3 & 7.141 & 0.518 & 0.698 \\
\hline Deviation & 41.326 & 3 & & \\
\hline Total & 22094.337 & 16 & & \\
\hline
\end{tabular}

\section{Table 9}

(i) The presentation of Tables and the formatting of text in the online proof do not match the final output, though the data is the same. To preview the actual presentation, view the Proof.

Orthogonal test results of multivariate analysis of variance of $l_{s}$ for hollow unit block structure.

\begin{tabular}{|l|l|l|l|l|l|}
\hline Variance source & Sum of square & Degree of freedom & Mean square & F value & P value \\
\hline Length $(\mathrm{cm})$ & 22.872 & 3 & 7.624 & 4.982 & 0.110 \\
\hline Width $(\mathrm{cm})$ & 24.440 & 3 & 8.147 & 5.324 & 0.102 \\
\hline & & & & \\
\hline
\end{tabular}




\begin{tabular}{|l|l|l|l|l|l|}
\hline Side walls thickness (cm) & 10.575 & 3 & 3.525 & 2.303 & 0.255 \\
\hline Bottom plate thickness (cm) & 1.218 & 3 & 0.406 & 0.265 & 0.848 \\
\hline Deviation & 4.591 & 3 & & \\
\hline Total & 237.843 & 16 & & \\
\hline
\end{tabular}

Based on Tables 8 and 9, it is suggested that all four factors were insignificant to $l_{s}$, meanwhile the structure width was the only significant factor to $\sigma_{m}$. That means the size of the structure which was perpendicular to the vehicle driving direction becomes the most important to analyse the mechanical properties for hollow unit block structure. This becomes remarkable to guide the design of the hollow unit block structure by paying more attention on the structure width when considering optimum structural mechanical properties of a PV unit block in the future work. Furthermore, the results based on the influence of bottom plate thickness were found similar to [18], which can be concluded it was insignificant to the mechanical properties of the hollow structure. Conversely, the conclusions about the significance of the side walls thickness and the structure length were opposite, which due to the different methods of analysis were employed. In the reference [18], they used the method of control variates, but the orthogonal analysis was introduced in this study.

\subsection{Feasibility of ABS}

In terms of the material for unit block body, the maximum Mises stress of ABS for the two structures are 5.74 $\mathrm{MPa}$ and 12.16 $\mathrm{MPa}$, respectively, which are much less than ABS allowable stress $24.5 \mathrm{MPa}$ [41]. Therefore, ABS satisfies the requirement of the mechanical properties for PV unit block (Fig. 6).

\section{Fig. 6}

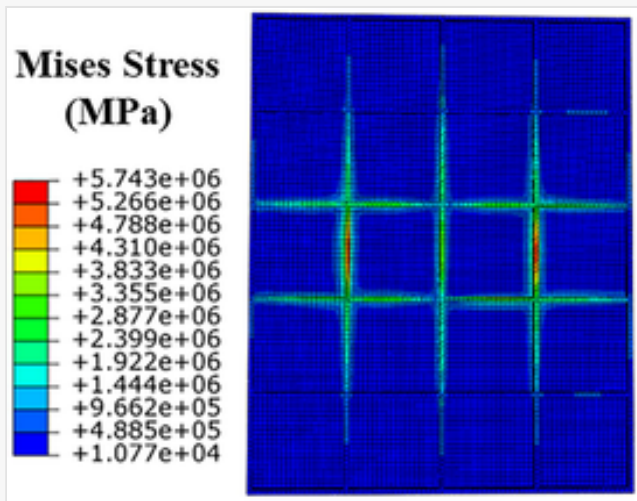

(a)

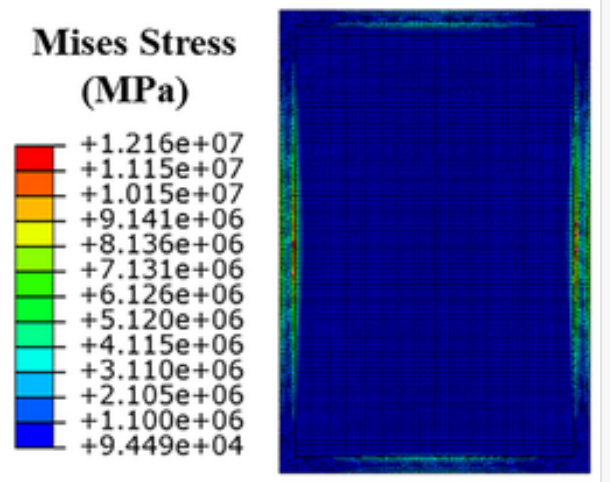

(b)

The maximum stress of ABS for (a) grid unit block and (b) hollow unit block.

\section{Conclusion and future work}

In this study, $\sigma_{m}$ and $l_{s}$ were considered as the indexes to discuss the mechanical properties for two different unit block structures of PV pavement, grid unit block and hollow unit block. Based on the ABAQUS 
simulation results and the orthogonal test design, the mean analysis and variance analysis were conducted. The optimal combination of various parameters for each structure and the significance of each factor towards the structural mechanical properties were ranked and obtained. Based on the findings, the main conclusions are drawn as follows.

(1) The best combination for each structure is:

(i) For the grid unit block structure: $120 \mathrm{~cm}$ length $\times 120 \mathrm{~cm}$ width $\times 8$ cm-thickness bottom plate $\times 2 \mathrm{~cm}$-thickness grid

(ii) For the hollow unit block structure: $-60 \mathrm{~cm}$ length $\times 60 \mathrm{~cm}$ width $\times 6 \mathrm{~cm}$-thickness bottom plate $\times 10 \mathrm{~cm}$-thickness side walls

(2) The rank of significance for various factors for each structure is:

(i) For the grid unit block structure: bottom plate thickness $>$ grid thickness $>$ width $>$ length

(ii) For the hollow unit block structure: only width is significant to $\sigma_{m}$, wherein, the length is parallel to the vehicle driving direction, and the width is perpendicular to the vehicle driving direction

(3) Base on the $l_{s}$ and $\sigma_{m}$ simulation result, the two structures have shown dissimilar mechanical response, which also suggested that the adaptation of grid unit block structure for a PV pavement is better than the adaptation of hollow unit block structure;

(4) In this study, the ABS was used as the material for the unit block and the results have indicated it satisfies the requirement of the mechanical properties.

From the conclusion, it is recommended for the future studies to pay more attention on determining the grid unit block structure which can provide better mechanical properties. The significance of the different factors should be thoroughly considered during the initial design stage of PV unit block structures.

As this study only considered the mechanical properties of the PV unit block structures, the future work will integrate the thermal impacts as well as the efficiency of its electricity generation.

\section{Authors Contributions}

Bochao Zhou and Jianzhong Pei conceived of the presented idea. Jianzhong Pei and Ben Richard Hughes supervised the project. Bochao Zhou performed the analytic calculations and performed the numerical simulations. Bochao Zhou took the lead in writing the manuscript, Diana SNM Nasir and Jiupeng Zhang contributed to improving the language and organization. All authors discussed the results and provided critical feedback and helped shape the research, analysis, and manuscript.

\section{Declaration of Competing Interest}


The authors declare that they have no known competing financial interests or personal relationships that could have appeared to influence the work reported in this paper.

\section{Acknowledgments}

Funding: This study was supported by the National Key R\&D Program of China (Nos. 2018YFE010380), the National Natural Science Foundation of China (Nos. 51978068), the Department of Science \& Technology of Shaanxi Province (Nos. 2017KCT-13), China Postdoctoral Science Foundation (Nos. 2017 M620434) and the Special Fund for Basic Scientific Research of Central College of Chang'an University (Nos. 310821153502 and 310821173501). The authors gratefully acknowledge their financial support.

\section{References}

The corrections made in this section will be reviewed and approved by journal production editor.

[1] Papadimitriou C.N., Psomopoulos C.S., Kehagia F., A review on the latest trend of Solar Pavements in Urban Environment, Energy Procedia 157 (2019) 945-952, doi:10.1016/j.egypro.2018.11.261.

[2] F. Kehagia, S. Mirabella, C.S. Psomopoulos, Solar pavement: a new source of energy, in: Bituminous Mixtures and Pavements VII: Proceedings of the 7th International Conference' Bituminous Mixtures and Pavements' (7ICONFBMP), June 12-14, 2019, CRC Press, Thessaloniki, Greece, p. 441.

[3] Li J., Xiao F., Zhang L., Amirkhanian S.N., Life cycle assessment and life cycle cost analysis of recycled solid waste materials in highway pavement: a review, J. Cleaner Prod. (2019), doi:10.1016/j.jclepro.2019.06.061.

[4] Silva R.V., de Brito J., Lynn C.J., Dhi R.K., Environmental impacts of the use of bottom ashes from municipal solid waste incineration: a review, Resour. Conserv. Recycl. 140 (2019) 23-35, doi:10.1016/j.resconrec.2018.09.011.

[5] Li R., Yu Y., Zhou B., Guo Q., Li M., Pei J., Harvesting energy from pavement based on piezoelectric effects: Fabrication and electric properties of piezoelectric vibrator, J. Renew. Sust. Energy 10 (5) (2018), doi:10.1063/1.5002731 054701.

[6] Ding G., Zhao X., Wang J., Xu C., Vibration energy harvesting from roads under traffic loads, Road Mater. Pavement Design (2018) 1-20, doi:10.1080/14680629.2018.1527719.

[7] Tahami S.A., Gholikhani M., Nasouri R., Dessouky S., Papagiannakis A.T., Developing a new thermoelectric approach for energy harvesting from asphalt pavements, Appl. Energy 238 (2019) 786-795, doi:10.1016/j.apenergy.2019.01.152. 
[8] Jiang W., Xiao J., Yuan D., Lu H., Xu S., Huang Y., Design and experiment of thermoelectric asphalt pavements with power-generation and temperature-reduction functions, Energy Build. 169 (2018) 39-47, doi:10.1016/j.enbuild.2018.03.049.

[9] H. Jiang, Y. Cen, X. Zha, Q. Zhang, Current Situation and Development Trend of Solar Pavement Technology. DEStech Transactions on Environment, Energy and Earth Sciences (epe), 2018.

[10] Ma T., Yang H., Gu W., Li Z., Yan S., Development of walkable photovoltaic floor tiles used for pavement, Energy Convers. Manage. 183 (2019) 764-771, doi:10.1016/j.enconman.2019.01.035.

[11] Pei J., Zhou B., Lyu L., e-Road: the largest energy supply of the future?, Appl. Energy 241 (2019) 174-183, doi:10.1016/j.apenergy.2019.03.033.

[12] Zhou B., Pei J., Xue B., Guo F., Wen Y., Zhang J., et al., Solar/road from 'forced coexistence' to ‘harmonious symbiosis', Appl. Energy 255 (2019), doi:10.1016/j.apenergy.2019.113808 113808.

[13] S.D. Brusaw, J.A. Brusaw, Solar Roadway Pane. U.S. Patent Application No. 29/452,723, 2014.

[14] How 'Solar Roadways' plans to create smart roads to produce clean energy and save lives and money. Available from: $<$ https://www.techrepublic.com/article/how-solar-roadways-plans-to-crea te-smart-roads-to-produce-clean-energy-and-save-lives-and-money/>. Accessed on 7 August, 2019.

[15] Northmore A.B., Tighe S.L., Developing innovative roads using solar technologies Edmonton, Canada, Can. Soc. Civil Eng. 2 (2012) 1348-1355.

[16] Northmore A.B., Canadian solar road panel design: a structural and environmental analysis[D], University of Waterloo, 2014.

[17] Northmore A.B., Tighe S.L., Performance modelling of a solar road panel prototype using finite element analysis, Int. J. Pavement Eng. 17 (2016) 449-457, doi:10.1080/10298436.2014.993203.

[18] Zha X., Zhang C., Wu Z., Zhang Q., Mechanical analysis and model preparation for hollow slab element of solar pavement, Acta Energiae Solaris Sin. 37 (2016) 136-141.

[19] Maghool F., Arulrajah A., Du Y.J., Horpibulsuk S., Chinkulkijniwat A., Environmental impacts of utilizing waste steel slag aggregates as recycled road construction materials, Clean Technol. Environ. Policy 19 (4) (2017) 949-958, doi:10.1007/s10098-016-1289-6.

[20] Zhou B., Pei J., Lv L., Zhang J., Qiu J., Design and evaluation of glassphalt mixtures by using recycled waste cullet, J. Southeast Univ. (English Edition) 35 (2) (2019) 198-205, doi:10.3969/j.issn.1003-7985.2019.02.009.

[21] P. Duggal, A.S. Shisodia, S. Havelia, K. Jolly, Use of waste plastic in wearing course of flexible pavement, in: Advances in Structural Engineering and Rehabilitation, Springer, Singapore, 2020, pp. $177-187$. 
[22] Patrick ten Brink, Jean-Pierre Schweitzer, Emma Watkins, Charlotte Janssens, Michiel De Smet, Heather Leslie, François Galgani, Circular economy measures to keep plastics and their value in the economy, avoid waste and reduce marine litter. Economics Discussion Papers, No 2018-3, Kiel Institute for the World Economy. Available from $<\underline{\text { http://www.economics-ejournal.org/econo }}$ mics/ discussionpapers/2018-3>.

[23] I.R. Sofi, J. Manzoor, R.A. Bhat, R. Munvar, Plastic pollution and the ecological impact on the aquatic ecosystem, in: Handbook of Research on Environmental and Human Health Impacts of Plastic Pollution, IGI Global, 2020, pp. 80-93.

[24] Sci. Adv. 3 (7) (2017) e1700782, doi:10.1126/sciadv.1700782.

[25] Zhou H., Long Y.Q., Meng A.H., et al., Thermogravimetric characteristics of typical municipal solid waste fractions during co-pyrolysis, Waste Manage. 38 (1) (2015) 194-200, doi:10.1016/j.wasman.2014.09.027.

[26] Wang W., Themelis N.J., Sun K., Bourtsalas A.C., Huang Q., Zhang Y., Wu Z., Current influence of China's ban on plastic waste imports, Waste Disposal Sust. Energy 1 (1) (2019) 67-78.

[27] Plastics Europe, 2016. Plastics - The Facts 2016. An analysis of European Plastics Production, Demand and Waste Data, Plastics Europe, Brussels.

[28] How many kinds of plastic are there? What are the common plastic materials? Where did he end up? Available from: $\quad$ https://www.huanbao-world.com/a/zixun/2018/0611/20731.html $>$. Accessed on 7 August 2019.

[29] A. Charles, P.M. Bassan, T. Mueller, A. Elkaseer, S.G. Scholz, On the assessment of thermomechanical degradability of multi-recycled ABS polymer for 3D printing applications, in: International Conference on Sustainable Design and Manufacturing, Springer Singapore, 2019, pp. 363-373.

[30] Leite M., Varanda A., Ribeiro A.R., Silva A., Vaz M.F., Mechanical properties and water absorption of surface modified ABS 3D printed by fused deposition modelling, Rapid Prototyping J. 24 (1) (2018) 195-203, doi:10.1108/RPJ-04-2016-0057.

[31] Xia W., Xu T., Wang H., Thermal behaviors and harmful volatile constituents released from asphalt components at high temperature, J. Hazard. Mater. 373 (2019) 741-752, doi:10.1016/j.jhazmat.2019.04.004.

[32] Ding X., Ma T., Gu L., Zhang Y., Investigation of surface micro-crack growth behaviour of asphalt mortar based on the designed innovative mesoscopic test, Mater. Des. 185 (2020) 108238, doi:10.1016/j.matdes.2019.108238.

[33] Zhang Y., Ma T., Ling M., Huang X., Mechanistic sieve size classification of aggregate gradation by characterizing load carrying capacity of inner structures, J. Eng. Mech. 145 (9) (2019) 
4019069, doi:10.1061/(ASCE)EM.1943-7889.0001640.

[34] Ding X., Ma T., Huang X., Discrete-element contour-filling modeling method for micro-and macro- mechanical analysis of aggregate skeleton of asphalt mixture, J. Transportation Eng. Part B: Pavements 145 (1) (2019) 4018056, doi:10.1061/JPEODX.0000083.

[35] Ma T., Zhang D., Zhang Y., Wang S., Huang X., Simulation of wheel tracking test for asphalt mixture using discrete element modelling, Road Mater. Pavement Design 19 (2) (2018) 367-384, doi:10.1080/14680629.2016.1261725.

[36] Zhang Y., Ma T., Ling M., Zhang D., Huang X., Prediction of dynamic shear modulus of asphalt mastics by using the discretized element simulation and reinforcement mechanisms, J. Mater. Civ. Eng. 31 (8) (2019) 4019163, doi:10.1061/(ASCE)MT.1943-5533.0002831.

[37] Zhu X., Dai Z., Chen F., Pan X., Xu M., Using the visual intervention influence of pavement marking for rutting mitigation-Part II: visual intervention timing based on the finite element simulation, Int. J. Pavement Eng. 20 (5) (2019) 573-584, doi:10.1080/10298436.2017.1316646.

[38] Zeng J., Mechanical analysis of hollow slab pavement structure, University of Science and Technology, Changsha, 2013.

[39] A.M.C. Souza, M.C. Cucchiara, Mechanical properties of virgin ABS/post-consumer ABS blends, in: AIP Conference Proceedings, 1981 (1) (2018) 020179. AIP Publishing, doi: $10.1063 / 1.5046041$.

[40] Cai L., Experimental Research on Structural Model Of Hollow Slab for Solar Energy Pavement, University of Science and Technology, Changsha, 2014.

[41] J. Wu, C. Zhao, Y. Liu, S. Ma, Mechanical analysis of a customized hand orthosis based on 3D printing, in: International Workshop of Advanced Manufacturing and Automation, 2017, September, Springer, Singapore, pp. 501-508, doi: 10.1007/978-981-10-5768-7_53.

\section{Highlights}

- The research considered two types of PV pavement unit block structure.

- The effects of four factors on the mechanical properties were analyzed.

- Orthogonal test was used to obtain 16 sets of experiment for each structure.

- The significance of each factor for the two structures were ranked. 
